\title{
Electrochemical Methods for Speciation of Trace Elements in Marine Waters. Dynamic Aspects
}

\author{
A. M. Mota, ${ }^{* \dagger}{ }^{\dagger}$ J. P. Pinheiro, ${ }^{\ddagger}$ and M. L. Simões Gonçalves ${ }^{\dagger}$ \\ ${ }^{\dagger}$ CQE, Instituto Superior Técnico, Technical University of Lisbon, Av. Rovisco Pais, 1049-001 Lisboa, Portugal \\ ${ }^{\ddagger}$ IBB/CBME, Department of Chemistry and Pharmacy, Faculty of Sciences and Technology, University of Algarve, 8005-139 Faro, \\ Portugal
}

\begin{abstract}
The contribution of electrochemical methods to the knowledge of dynamic speciation of toxic trace elements in marine waters is critically reviewed. Due to the importance of dynamic considerations in the interpretation of the electrochemical signal, the principles and recent developments of kinetic features in the interconversion of metal complex species will be presented. As dynamic electrochemical methods, only stripping techniques (anodic stripping voltammetry and stripping chronopotentiometry) will be used because they are the most important for the determination of trace elements. Competitive ligand exchange-adsorptive cathodic stripping voltammetry, which should be considered an equilibrium technique rather than a

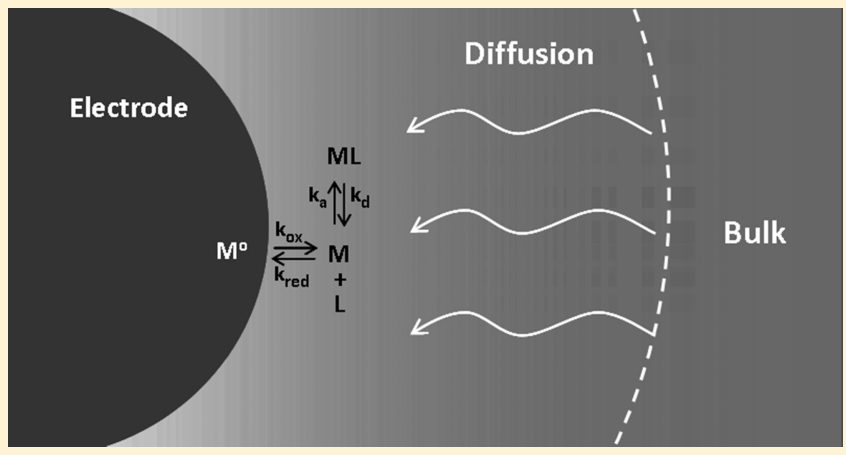
dynamic method, will be also discussed because the complexing parameters may be affected by some kinetic limitations if equilibrium before analysis is not attained and/or the flux of the adsorbed complex is influenced by the lability of the natural complexes in the water sample. For a correct data interpretation and system characterization the comparison of results obtained from different techniques seems essential in the articulation of a serious discussion of their meaning.
\end{abstract}

\section{INTRODUCTION}

Speciation is the key factor to understand the transport, bioavailability, and toxicity of metal ions. Because aquatic systems are practically never at chemical equilibrium, dynamic speciation is necessary to understand the nonequilibrium properties. On the other hand, in speciation models where toxicity is mainly due to the free metal ion permeability through the biological membranes, the fraction responsible for the bioavailability of metal ions includes not only the free metal ion but also the complexes that are mobile and can dissociate during the time scale of adsorption/transport. Detailed analysis of the fluxes and kinetics involved in metal biouptake revealed that the diffusive transport and the rate of complex dissociation may contribute to the overall process. ${ }^{1-3}$ Free ion activity (FIAM) and biotic ligand (BLM) models have been increasingly used to describe the relationships between chemical speciation and biological availability, ${ }^{4,5}$ but in their simplest form they are based on the concept that the formation of any complex in solution will reduce trace metal uptake and, thus, reduce metal bioavailability, neglecting dynamic aspects of interconversion of species and the possibility of some inert complexes to be transported directly through the biological cell membrane.

Dynamic voltammetric techniques, well suited to determine the labile fraction at different time scales (Figure 1 ), ${ }^{3}$ can contribute for a better understanding of toxicity mechanisms

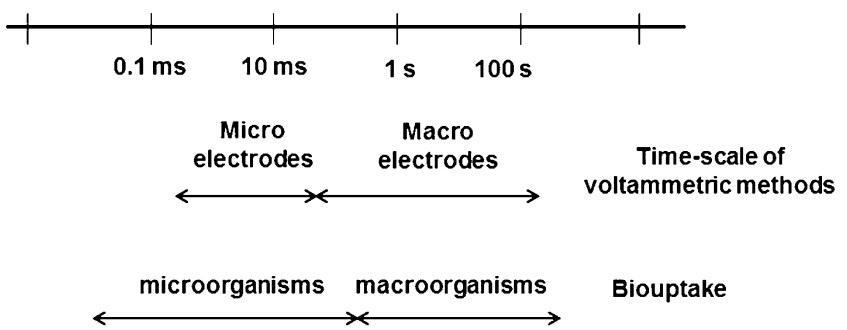

Figure 1. Comparison between the time scale of voltammetric methods and metal ion uptake by bioorganisms.

because under certain conditions it was found that labile metal correlates well with the toxic fraction of the metal ion. ${ }^{1}$

In this paper the principles and recent developments of kinetic features in the interconversion of metal complex species will be reviewed as well as the potentialities and drawbacks of stripping electrochemical methods applied to trace element speciation in marine waters, mainly oriented to the dynamic aspects of these techniques. Basically, two stripping techniques involving dynamic aspects, anodic stripping voltammetry and stripping chronopotentiometry, have been used in saline waters

Special Issue: Herman P. van Leeuwen Festschrift

Received: December 25, 2011

Revised: April 29, 2012

Published: April 29, 2012 
for the determination of the labile fraction as well as of the stability and kinetic parameters. Competitive ligand exchangeadsorptive cathodic stripping voltammetry, widely used in trace metal speciation in marine waters, will be also presented because some dynamic features may affect the voltammetric signal.

The examples given in this paper in the application of electrochemical methods to marine waters were oriented to copper, lead, cadmium, zinc, nickel, chromium, and arsenic speciation, because these elements have important toxic effects in the biota at low concentration levels.

\section{DYNAMIC SPECIATION BY ELECTROCHEMICAL METHODS}

Dynamic speciation modeling requires not only the knowledge of equilibrium parameters but also the kinetic features of the interconversion of metal complex species. Diffusion and/or kinetic fluxes of the various metal species in solution, both depending on the time scale of the technique and on the intrinsic characteristics of the complexing species, influence the voltammetric signal in dynamic techniques.

The concept of lability is used to describe the contribution of metal species to an overall flux toward a consuming interface based on the relative magnitudes of their diffusive (mass transport) and kinetic (dissociation) fluxes. ${ }^{2}$ Two extreme conditions can be described: ${ }^{1,6,7}$ (i) Complex species do not have time to dissociate/associate in the diffusion layer due to the slowness of the kinetic process and will not contribute to the signal (static system, inert complexes). In the presence of inert complexes the analytical signal (current) will be directly related to the free metal ion in the bulk solution. (ii) The rates of metal complex association/dissociation are high enough, so that the kinetic flux arising from dissociation of the complex into $\mathrm{M}$ in the diffusion layer is greater than the diffusion-limited flux, so that the complex species dissociate/associate in the diffusion layer (dynamic system). In this case two situations are possible: (a) The kinetic flux is much larger than the diffusive one. Thus the free metal ion will be in equilibrium with its complex forms all along the diffusion layer up to the electrode surface, and the total metal concentration will contribute to the signal (labile complexes). (b) Intermediate situations exist where the kinetic and diffusive fluxes are of the same order of magnitude. In this case the measured signal will be influenced by the kinetic characteristics (nonlabile complexes).

Besides the association/dissociation constant rates, the diffusion coefficient of complex species in the diffusion layer also affects the voltammetric signal in dynamic systems. The presence of macromolecular ligands as fulvic or humic material slows down the flux of dynamic metal ions due to lower diffusion coefficient of those ligands. For labile complexes a mean diffusion coefficient taking into account all the diffusing species should be considered. ${ }^{1,8}$ The species measured are those that are both mobile and labile for the given technique, and will include free metal ions and labile complexes in the size range up to a few hundreds of nanometers. ${ }^{9}$

Due to the importance of the dynamic considerations in the interpretation of the electrochemical signal, the development of the kinetic features in the interconversion of metal complex species will be reviewed in the next paragraphs.

2.1. Basic Eigen Mechanism. The kinetics of metal ion complexation in aqueous systems described by the Eigen mechanism $^{10}$ is composed of two essential steps: (i) the formation of a precursor outer-sphere complex, (ii) followed by the release of water from the inner sphere of the metal ion to form a coordination bond with the ligand:

$$
\begin{aligned}
& \mathrm{M}\left(\mathrm{H}_{2} \mathrm{O}\right)_{n}+\mathrm{L} \underset{k_{d}^{\text {os }}}{\stackrel{\mathrm{k}_{\mathrm{a}}^{\text {os }}}{\Leftrightarrow}} \mathrm{M}\left(\mathrm{H}_{2} \mathrm{O}\right)_{n} \cdot \mathrm{L} \\
& \mathrm{M}\left(\mathrm{H}_{2} \mathrm{O}\right)_{n} \cdot \mathrm{L} \underset{k_{\mathrm{d}}^{\text {is }}}{\stackrel{k_{\mathrm{s}}^{\text {is }}}{\Leftrightarrow}} \mathrm{M}\left(\mathrm{H}_{2} \mathrm{O}\right)_{n-1} \mathrm{~L}+\mathrm{H}_{2} \mathrm{O}
\end{aligned}
$$

where $k_{\mathrm{a}}^{\mathrm{os}}$ and $k_{\mathrm{d}}^{\mathrm{os}}$ are the formation and dissociation rate constants for the outer-sphere complex, $\mathrm{M}\left(\mathrm{H}_{2} \mathrm{O}\right)_{n} \cdot \mathrm{L} ; k_{\mathrm{a}}^{\text {is }}$ and $k_{\mathrm{d}}^{\text {is }}$ are the rate constants for the substitution of $\mathrm{H}_{2} \mathrm{O}$ by the monodentate ligand $\mathrm{L}$ in the inner-sphere complex and vice versa, respectively.

The rate-limiting step for the inner-sphere complex formation is the elimination of a water molecule from the inner hydration shell of $\mathrm{M}\left(\mathrm{H}_{2} \mathrm{O}\right)_{n}$. Its rate constant is usually denoted as $k_{\mathrm{w}}$ and thus $k_{\mathrm{a}}^{\text {is }}=k_{\mathrm{w}}$. The value of $k_{\mathrm{w}}$ is independent of the ligand and the values for common metals can be found in the literature, ${ }^{11}$ ranging from $7 \times 10^{9} \mathrm{~s}^{-1}$ for $\mathrm{Pb}^{2+}$ to $5 \times 10^{-7}$ $\mathrm{s}^{-1}$ for $\mathrm{Cr}^{3+}$.

Until recently it was accepted that $k_{\mathrm{w}}$ was the rate limiting step of the overall process of complex formation, leading to

$$
k_{\mathrm{a}}=K^{\mathrm{os}} k_{\mathrm{w}}
$$

where $k_{\mathrm{a}}$ is the rate constant of the complex formation and $K^{\text {os }}$ the stability constant of the outer-sphere complex (reaction 1). $K^{\mathrm{os}}$ depends on the primary Coulombic energy between the metal and the ligand, and on the energy for the screening effect due to the presence of electrolyte. ${ }^{12,13}$ Its value dependent on the charges of $\mathrm{M}$ and $\mathrm{L}$ and on the ionic strength. ${ }^{14}$

The basic Eigen mechanism was kept virtually unchanged in the electroanalytical field since the introduction of the first lability criteria ${ }^{15}$ (Figure 2) until $2005 .{ }^{16}$ With the advent of the

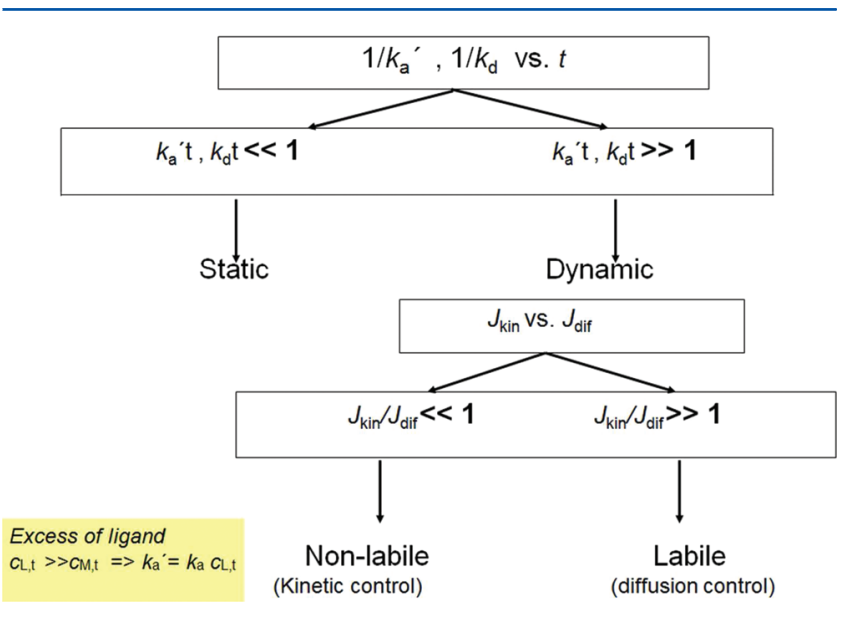

Figure 2. Dynamic and lability criterium. $c_{\mathrm{L}, \mathrm{t}}$ and $c_{\mathrm{M}, \mathrm{t}}$ are the total ligand and metal concentrations, respectively; $J_{\text {dif }}$ and $J_{\text {kin }}$ are the diffusion and kinetic fluxes, respectively.

stripping chronopotentiometry in the early 2000s, and especially the scanned deposition potential mode in 2003, the direct determination of the kinetic parameters using that technique clearly showed that the basic Eigen mechanism could not explain more involved systems like the metal ion complexation in colloidal systems and/or with multidentate ligands.

Since 2005, the development of the kinetic features in the interconversion of metal complex species has been based on 
three different aspects: (i) effect of ligand protonation; (ii) ion binding with a strongly charged macromolecule (e.g., fulvic and humic acids); (iii) heterogeneous ligand and charge distribution in colloidal dispersions.

2.2. Impact of Ligand Protonation on Metal Dynamic Speciation. In 2007, van Leeuwen et al. ${ }^{17}$ addressed for the first time the impact of ligand protonation on metal speciation dynamics. The stability of the outer-sphere complex for multidentate ligands containing several protonated sites was thoroughly investigated. The ligand protonation impact on the complexation kinetics of higher-order complexes $\left(\mathrm{ML}_{2} \cdots \mathrm{ML}_{n}\right)$ was analyzed and expressions including contributions of all outer-sphere complexes to the rate of complex formation were derived. ${ }^{18}$ The effect of the ligand protonation was also investigated in the formation/dissociation kinetics of inner sphere metal complexes. ${ }^{19}$ Although all protonated forms of the ligand contribute to the formation of the precursor outersphere complexes, only the sufficiently stable ones effectively contribute to the overall rate of inner-sphere complex formation, even if they are minor components in bulk solution. This result highlights the importance of distinguishing between the thermodynamically predominant species versus the kinetically relevant ones in considerations of dynamic speciation analysis.

2.3. Metal Ion Binding with Charged Macromolecules and in Colloidal Dispersions. Buffle et al. ${ }^{20}$ pointed out that in aquatic environmental systems a number of natural ligands with small diffusion coefficients and highly negative charged (e.g., fulvic acids) might lead to lower $k_{\mathrm{a}}^{\text {os }}$ and $k_{\mathrm{d}}^{\text {os }}$ values. Under such conditions, the formation/dissociation of the outer-sphere complex by diffusion of $\mathrm{M}$ and $\mathrm{L}$ may become the rate-limiting step on the complex formation.

In steady state the overall rate constant, $k_{a}$, is given by ${ }^{21}$

$$
k_{\mathrm{a}}=\frac{k_{\mathrm{a}}^{\mathrm{os}} k_{\mathrm{w}}}{k_{\mathrm{d}}^{\mathrm{os}}+k_{\mathrm{w}}}
$$

When $k_{\mathrm{d}}^{\mathrm{os}} \gg k_{\mathrm{w}}, k_{\mathrm{a}} \approx K^{\mathrm{os}} k_{\mathrm{w}}$. This corresponds to the situation where the loss of the water molecule from the inner hydration shell is the rate limiting step (Eigen mechanism).

In general, three conditions should occur to obtain values of $k_{\mathrm{d}}^{\text {os }}$ similar to (or even smaller than) $k_{\mathrm{w}}$ : (i) metal ions with very fast dehydration $\left(k_{\mathrm{w}}\right.$ values in the upper range, e.g., $\mathrm{Pb}^{2+}, \mathrm{Hg}^{2+}$, $\mathrm{Cu}^{2+}$ ); (ii) ligands with high negative charges; (iii) ionic strength rather low.

Pinheiro et al. ${ }^{16}$ pointed out that, in colloidal systems, the ligands are localized within the vicinity of the particles, whereas in true solutions the ligand distribution is taken as homogeneous over the solution volume (Figure 3). In this

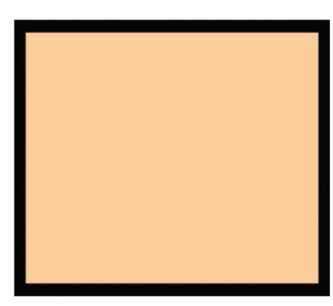

true solution

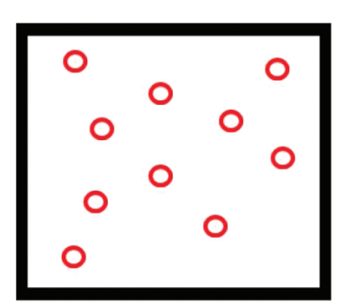

colloidal dispersion
Figure 3. Homogeneous ligand solution versus colloidal dispersion of ligands. In the colloidal dispersions the ligands are solely present within the volume of the colloidal particles. case the local diffusion-controlled transport of metal ions to/ from the particle must be taken into account. For a hard particle where all ligands lay at the surface, the apparent rate constant of complex formation (and dissociation) should be given by

$$
k_{\mathrm{a}}^{* \prime}=k_{\mathrm{a}}^{\prime} /\left(1+k_{\mathrm{a}}^{\prime}\left(4 \pi a D_{\mathrm{M}} c_{\mathrm{p}}\right)^{-1}\right)
$$

where $c_{\mathrm{p}}$ is the particle number density and $a$ is the particle radius. The prime denotes the situation of excess ligand (total ligand concentration $c_{\mathrm{L}, \mathrm{t}}$ much larger than the total metal concentration $c_{\mathrm{M}, \mathrm{t}}$ so that $\left.c_{\mathrm{L}} \cong c_{\mathrm{L}, \mathrm{t}}\right)$, where $k_{\mathrm{a}}^{\prime}=k_{\mathrm{a}} c_{\mathrm{L}, \mathrm{t}}$.

For a colloidal ligand dispersion, the apparent rate constant, $k_{\mathrm{a}}^{* \prime}$, has two limiting values: (i) a kinetic limit for $k_{\mathrm{a}}^{\prime}\left(4 \pi a D_{\mathrm{M}} c_{\mathrm{p}}\right)^{-1} \ll 1$, at which $k_{\mathrm{a}}^{* \prime}=k_{\mathrm{a}}^{\prime}$. This situation occurs for metals with relatively low formation rate constants (low $k_{\mathrm{w}}$ ) and/or systems with small particles; (ii) a diffusive limit for $k_{\mathrm{a}}^{\prime}\left(4 \pi a D_{\mathrm{M}} c_{\mathrm{p}}\right)^{-1} \gg 1$, at which $k_{\mathrm{a}}^{* \prime}=4 \pi a D_{\mathrm{M}} \mathcal{c}_{\mathrm{p}}$. This limit is expected for metals with relatively high $k_{\mathrm{w}}$ values and/or relatively large colloidal particles.

Furthermore, colloidal dispersions are different from small ligands due to the nonhomogeneous distribution of binding sites and charges, possible variations in size and/or shape originating variations in their mobility, and chemical heterogeneity of the binding sites. These differences lead to a series of extensions and variations of the effective rate constant of association (eq 5).

Buffle et al. $^{22}$ developed a similar equation for aggregates (taken as porous particles) and van Leeuwen ${ }^{23}$ demonstrated the applicability of Eigen complexation kinetics to metal sorption at surfaces.

Duval et al. ${ }^{24}$ proposed a theory that takes into account bulk dispersions of core-shell colloidal ligands ranging from hard to porous particles. Later ${ }^{25}$ he incorporated the impact of the electric double layer (EDL) field and inhomogeneous site distribution inside the particle. Still in 2009, Duval and Qian ${ }^{26}$ studied the effect of subjecting the system to a steady-state laminar flow condition. In this case mass transfer and subsequent complexation of metal species within the reactive, permeable particle shell are governed by the interplay between (i) convective-diffusion of free metal ions $\mathrm{M}$ within and around the shell where ligands $\mathrm{L}$ are distributed, and (ii) kinetics of $\mathrm{ML}$ complex formation/dissociation in the shell.

A comprehensive review of the basic principles of metal ion formation/dissociation kinetics of metal ions with complexants of different size ranges was presented in 2009 by van Leeuwen and Buffle. ${ }^{21}$ Finally, in 2011, van Leeuwen et al. ${ }^{27}$ presented a theoretical discussion describing the formation and dissociation rate constants for metal ion binding by soft colloidal complexants, where they compared the effects of the electrostatic potential and colloid radius on the overall complexation reaction for simple ligands.

Although our understanding of the kinetics of metal ion binding with a variety of ligands has increased significantly in the last six years, a series of issues still need to be developed, for instance accounting for the effect of electric double-layer field outside and inside the particle, investigating if the diffusion coefficient of the metal ion in the colloidal body may change the kinetics and evaluate the rate of establishment of the equilibrium potential within the particle.

2.4. Lability Criterion. If the metal ion interaction with the ligands is dynamic in a voltammetric technique, then it becomes crucial to determine whether the complexes are labile 
or nonlabile. The degree of lability should be known a priori in techniques like ASV, because it will determine the equations used in the analysis of the experimental results.

It is not surprising to find that the development of lability criteria has been one of the first and most investigated aspects of dynamic speciation. ${ }^{15,28}$ It is important to emphasize that the "labile fraction" is ill-defined because it depends on the time scale of the technique. Hence, different techniques (or conditions within a technique) can "see" different "labile fractions". 3

In 2001, van Leeuwen ${ }^{2}$ reviewed the concept of lability of metal complexes and pointed out that it had been investigated since the 1950s by Heyrovsky, Brdicka, Koutecky, Koryta, and other members of the Czech school of electrochemistry (references therein), within the polarographic methods. Readers are referred to that manuscript for details of the evolution of the concepts up to 2001.

Still in 2001, Galceran et al., ${ }^{29}$ discussing the influence of the size of a microelectrode on the relative importance of the diffusional and kinetic contributions to the voltammetric current, introduced the useful concept of lability degree $(\xi)$ (1 fully labile, 0 nonlabile):

$$
\xi=\left(J_{\text {kinetic }}-J_{\text {inert }}\right) /\left(J_{\text {labile }}-J_{\text {inert }}\right)
$$

where $J_{\text {kinetic }} J_{\text {labile, }}$ and $J_{\text {inert }}$ are the kinetic, labile, and free metal fluxes respectively. Molina et al., in $2009^{30}$ and 2010, ${ }^{31}$ proposed more accurate expressions of $\xi$ for spherical sensors based on the diffusive-kinetic steady-state (dkss) approximation. Simple time-dependent expressions for the surface metal flux, the lability degree and the half-wave potential are presented, valid for any value of the ratio of concentrations at the surface and the sensor radius.

In 2002, van Leeuwen et al. ${ }^{32}$ evaluated the KouteckyKoryta approximation which assumes a discontinuous transition in the concentration profiles from nonlabile to labile behavior, i.e., a spatial separation of a reaction layer (from the surface of the electrode to the reaction layer thickness, $\mu$ ) and a equilibrium layer (from $\mu$ until the diffusion layer thickness, $\delta)$. The results indicate that the approximation is very good in the complete kinetic range from nonlabile to labile complexes, as long as the kinetic flux is computed from the effective concentration of the complex in the reaction layer. This result is extremely important because it allows a much simpler computation of dynamic currents in electrochemical experiments.

A significant advance in lability studies arose with the development of scanned stripping chronopotentiometry (SSCP) in the early 2000s. The relatively simple mathematical framework of this technique, together with the far-reaching validity of the Koutecky-Koryta approximation allowed van Leeuwen and Town ${ }^{33}$ to derive a rigorous kinetic expression for the full SSCP wave. In parallel, Pinheiro and van Leeuwen ${ }^{34}$ presented an experimental method to assess the lability of metal complexes (where the ligand concentration is much larger than the metal ion) by comparing the stability parameter $K^{\prime}$ computed from variations in potential shift and limiting wave height in absence and presence of complexing ligands. It was demonstrated that this approach is a sensitive indicator of lability in the analysis of cadmium and lead binding by carboxylated nanospheres.

The development of the theory of metal speciation in colloidal dispersions ${ }^{16}$ effectively modifies the finite rates of association/dissociation of the colloidal metal complexes, thus invoking the consideration of two basic dynamic criteria: the association/dissociation kinetics of the volume complexation reaction (the "dynamic" criterion) and the interfacial flux of free metal to a macroscopic surface due to dissociation of complex species (the "lability" criterion). Pinheiro et al. demonstrated ${ }^{35}$ that the conventional approach for homogeneous systems that assume a smeared-out ligand distribution, overestimates both the dynamics and the lability of metal complexes when applied to colloidal ligands. They also showed that the increase of lability with the particle radius, which is expected for homogeneous solutions, is moderated for colloidal dispersion in spherical microelectrodes and practically eliminated in planar electrodes. A lability criterion was developed for dynamic metal binding by colloidal ligands when convective diffusion is the dominant mode of mass transport. ${ }^{36}$ SSCP measurements of $\mathrm{Pb}$ (II) and $\mathrm{Cd}$ (II) binding to carboxylated latex core-shell particles were in good agreement with the predicted values. In that work it was especially important to verify that, due to the nature of the spatial distribution of the binding sites, the change in lability of a metal species with ligand concentration depends on whether this concentration is varied via manipulation of the $\mathrm{pH}$ (degree of protonation) or via the particle concentration. In the former case the local ligand density varies, whereas in the latter case it is constant producing different results in terms of lability behavior for the same total number of binding sites.

One of the most active areas of research regarding the complex lability has been the influence of the mixtures of ligands. This was addressed by Galceran et al. in $2003^{37}$ who developed a theoretical treatment taking into account all the dynamic properties of the mixture, including different mobilities and no restriction for the values of the association/ dissociation rate constants. The theory is valid for any geometry that can sustain steady-state flux. They found a lability degree for each 1:1 complex in terms of the surface concentrations leading to (i) a lability criterion specific for each complex in the mixture and (ii) the assessment of the relative contribution of each complex to the resulting flux. Directly following this work, Puy et al. $^{38}$ analyzed the voltammetric lability of a complex system, where a metal ion $M$ and a ligand $L$ form the species $\mathrm{ML}$ and $\mathrm{ML}_{2}$. They presented a rigorous numerical simulation of the problem and analytical solutions for the cases where $\mathrm{ML}$ $\rightarrow \mathrm{M}$ is the kinetically limiting step and the case where $\mathrm{ML}_{2} \rightarrow$ $\mathrm{ML}$ is the limiting one. On the basis of this work, van Leeuwen and Town $^{39}$ experimentally verified that the analytical expression for the lability of $\mathrm{ML}_{2}$ complexes, as determined by the rate of the final step $\mathrm{ML} \rightarrow \mathrm{M}$, was applicable for several metal-ligand systems at both conventional macroelectrode and microelectrode. The theory was generalized to a general 1:n metal/ligand stoichiometric ratio by Salvador et al. in $2006 .^{40}$

A notable evolution in the understanding of lability for metal ions in presence of ligand mixtures was presented in 2006 and 2007. ${ }^{41,42}$ As a consequence of the coupling of the association and dissociation processes for all complexes according to the competitive complexation reaction scheme, the lability of a given complex usually increases if another more labile complex is added into the system, whereas it decreases upon addition of a less labile one. The impact of the mixture effect on the metal flux depends at least on two main factors: the respective abundance of the metal species and the particular values of their lability degrees. For a mixture of many complexes, the change in the lability degree of a complex due to the mixture effect can be understood as a combination of the changes due to all of the complexes present. 


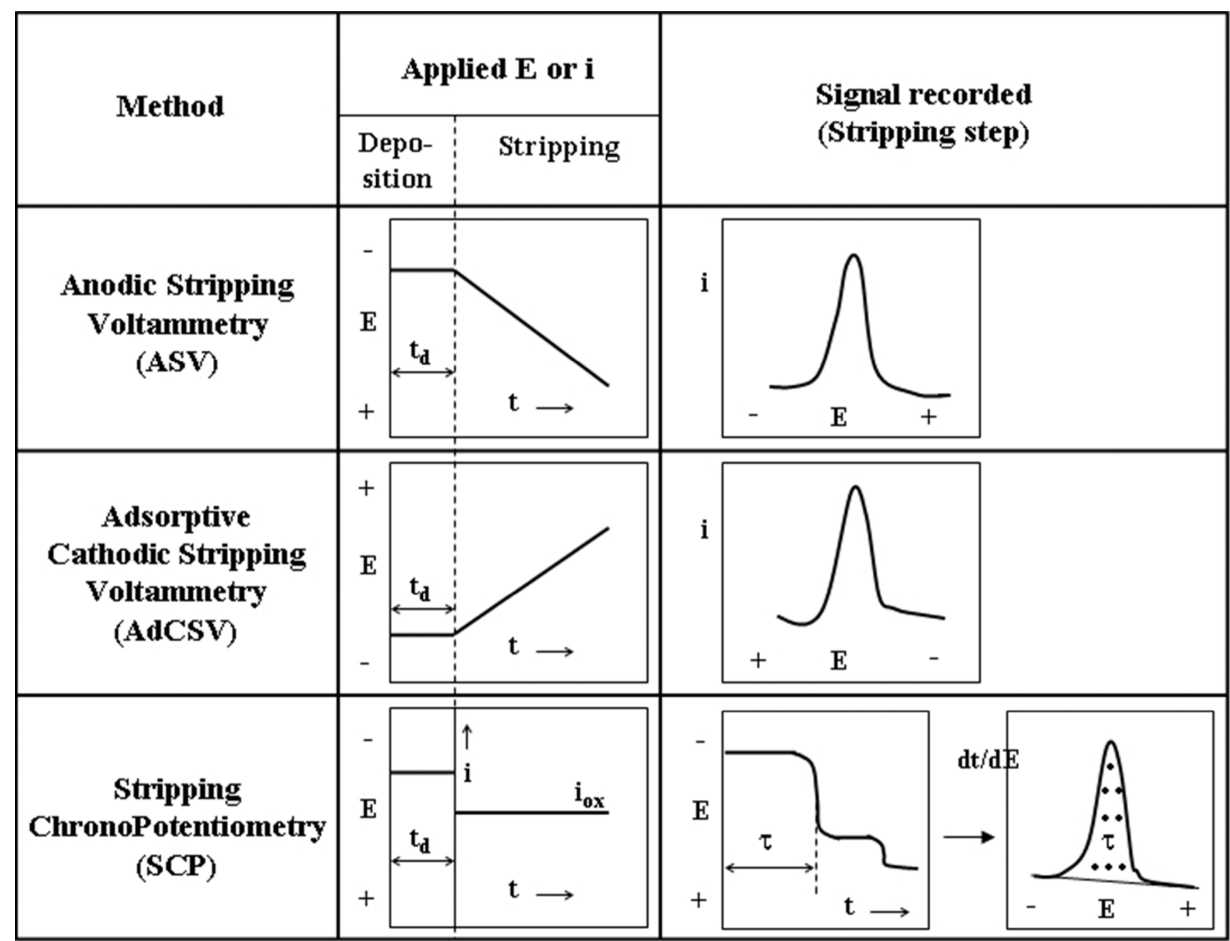

Figure 4. Stripping techniques used for trace element speciation in marine waters. $E$ is the potential, $t_{\mathrm{d}}$ is the deposition time, and $i$ is the current. The potential is usually modulated (differential pulse or square wave) in the stripping step of ASV and of AdCSV.

Buffle et al. first reconsidered the fundamentals of the reaction layer approximation and extended it to multiligand systems, where small and environmentally relevant ligands were considered. ${ }^{43-47}$ Metal fluxes at consuming interfaces can be determined using FLUXY, ${ }^{48}$ a computer code based on the reaction layer approximation under the assumptions of a ligand excess and equilibrium among successive complexes. FLUXY (available at http://www.unige.ch/cabe/dynamic/) enables quick computation and is applicable to natural ligands under many environmental conditions. Although each metal complex has its own reaction layer (so-called composite reaction layer), which results from the interplay of this particular complex with all the other complexes, the overall metal flux can be computed by assuming the existence of one single fictitious equivalent reaction layer thickness for the whole of the complexes. Flux enhancement might play a significant role in metal uptake in environmental or biological systems and should be considered in data interpretation.

In 2010 Pinheiro et al. $^{49}$ provided the first experimental evidence of the flux enhancement phenomena by using the voltammetric techniques AGNES and stripping chronopotentiometry applied to two systems: Cd/NTA/glycine and Cd/ NTA/citric acid. The flux measured in both cases was in good agreement with the flux computed for the global system, exhibiting maximum enhancement ratios above $20 \%$.

2.5. Stripping Techniques. For the determination of the labile fraction and complexing parameters of trace elements in marine waters, the potentialities and drawbacks of electrochemical techniques with stripping mode will be presented, because this mode is fundamental to achieve low detection limits in the determination of trace elements in seawaters (less than $\left.10^{-7} \mathrm{~mol} \mathrm{dm}^{-3}\right){ }^{50}$ Stripping techniques include two steps: the deposition step (accumulation) for a fixed period of time, to preconcentrate the analyte in the electrode, followed by the stripping step (quantification), involving the strip of the analyte into the solution. Basically, three stripping techniques, anodic stripping voltammetry, stripping chronopotentiometry and competitive ligand exchange-adsorptive cathodic stripping voltammetry have been used in marine waters for speciation purposes. The application of these techniques to trace metal speciation in environmental waters was recently reviewed by Pesavento et al., ${ }^{51}$ who commented on the relation between the electrochemical signal and the metal species which actually contribute to the current.

The two steps of each stripping technique are presented in Figure 4.

2.5.1. Anodic Stripping Voltammetry. Anodic stripping voltammetry (ASV) was one of the first and most widely used techniques for analysis of metal-organic complexation in seawater. It has been extensively applied to metals soluble in mercury, such as $\mathrm{Cu}, \mathrm{Pb}, \mathrm{Cd}$, and $\mathrm{Zn}$.

In the deposition step, a potential in the limiting current is applied and the metal ions in the labile fraction (free metal ion plus the metal ion from the ML that can be dissociated close to the electrode surface within the time scale of the technique) are electrochemically reduced and amalgamated in the mercury electrode. In the stripping step, the potential is scanned to more positive values and the signal (current versus potential), due to the metal oxidation into the solution, is measured (Figure 4).

For the determination of trace metal complexing parameters the sample is usually titrated with the metal ion at the natural $\mathrm{pH}$ to not disturb the medium. To keep the $\mathrm{pH}$ constant, most times buffers are added. To overcome the drawbacks of buffers (contamination and complexation) and of $\mathrm{O}_{2}$ degassing with pure nitrogen (which also removes $\mathrm{CO}_{2}$ resulting in $\mathrm{pH}$ changes), a mixture of nitrogen and $\mathrm{CO}_{2}$ is recommended under well controlled flow-rate, to keep the $\mathrm{pH}$ of the sample. ${ }^{52}$

The metal added to the sample must be left with the natural ligands until equilibration is attained, which may involve times as long as $1 \mathrm{~h}$ or higher, depending on the kinetics of 
complexation. It has been shown that in marine environments kinetics of trace metal complexation can be considerably retarded, mainly because of the competitive effect with calcium-magnesium cations. The slow dissociation rate of $\mathrm{Ca}$ and Mg-NOM complexes and competition with other trace elements are key factors that control the degree of trace metal complexation and the kinetics of its association with NOM. $^{53-55}$ Therefore, an equilibration time for complete complexation of added metal ion in each titration point (control of complexation kinetics) and an accumulation time (control of the sensitivity) are the key points to obtain reliable results free of artifacts. ${ }^{56}$

For inert complexes, the free metal ion concentration is determined from the peak current. In the presence of labile species, the complexing parameters can be obtained from peak potential $\left(E_{\mathrm{p}}\right)$, and if the diffusion coefficient of the metal complex is significantly lower than that of the metal ion, they can also be determined from the peak current $\left(i_{\mathrm{p}}\right) .{ }^{57}$ Detailed analysis of the shifts in the peak potential and of peak-current changes under different time scales of the technique should be performed beforehand to check the lability of the complex. ${ }^{1,50,57}$ Varying the diffusion layer thickness via different stirring rates of the solution or different rotation values of the electrode, different time scales are obtained and fractions of different lability may be measured.

The $i_{\mathrm{p}}$ decrease of the natural sample compared to the calibration plot should be carefully analyzed, because it may derive either from a slow kinetics of $\mathrm{ML}$ or from a low diffusion coefficient of the ML complex compared to the one of the metal ion $\left(D_{\mathrm{ML}}<D_{\mathrm{M}}\right){ }^{8}$ The free metal concentration determined from $i_{\mathrm{p}}$ will be different if inert complexes, or labile complexes with $D_{\mathrm{ML}}<D_{\mathrm{M}}$, are assumed. It is strongly recommended that each voltammetric complex titration is accompanied by a proper characterization of the kinetic features (and, if necessary, the diffusion coefficient) of the complex species. ${ }^{58} \mathrm{~A}$ large number of papers has estimated the free metal ion concentration (or the complexing capacity of marine waters) assuming that the complexes remain inert during the titration curve or if in the labile fraction all the complexes have $D_{\mathrm{ML}} \sim D_{\mathrm{M}}$. This should be reviewed on the basis of the strong evidence of metal ions in colloidal forms in marine environments. 59

For heterogeneous systems and labile systems, the information from $i_{\mathrm{p}}$ is not directly related with the one obtained from $E_{\mathrm{p}}$ in ASV. This is because $i_{\mathrm{p}}$ responds to the deposited metal, function of the metal complexation in the bulk solution, whereas $E_{\mathrm{p}}$ responds to the complexation at the electrode surface in the stripping step. Because the total metal ion in solution at the electrode surface is higher than in the bulk, weaker binding sites are being occupied, leading to lower complexing parameters determined from $E_{\mathrm{p}}$ compared to those calculated from $i_{\mathrm{p}}$.

Summarizing, measurements and data interpretation are sensitive to the ${ }^{50,57}$ (i) kinetics of dissociation of the various forms of complexed metal ions, (ii) hydrodynamic conditions (depending on the stirring rate or glassy carbon rotation rate), (iii) heterogeneity of the natural sample, (iv) ML diffusion coefficient if macromolecules including colloidal organic matter are present, (v) ligand concentration and surface effect (due to the absence of ligand excess at the electrode surface in the stripping step), and (vi) adsorption of organic matter on the electrode.
The influence of heterogeneity and absence of ligand excess in the stripping mode can be suppressed by exchanging ${ }^{60}$ or altering $^{61}$ the medium between the deposition and stripping steps. Because in most laboratories medium exchange is not a routine procedure, the alteration of the medium seems to be easier. In medium exchange the test solution is replaced before the stripping by a simple electrolyte. In that case information will be only obtained from $i_{\mathrm{p}}$ that exclusively depends on the parameters of the electrodeposition step. The major difficulty of this method lies on the complete medium exchange without perturbation of the electrode. To overcome this problem, an alternative strategy can be adopted on the basis of the addition of an excess of a strong, well-defined ligand after the deposition step. This method was applied mainly in the determination of copper in sea waters, using, e.g., ethylenediamine. The major drawback is that the stripping step should be performed only after reaching the equilibrium in solution (e.g., test solution allowed to stand overnight).

Speciation of $\mathrm{Cu}, \mathrm{Zn}, \mathrm{Pb}$, and $\mathrm{Cd}$ in environmental saline waters has been determined from ASV measurements by many authors. Copper has been the chosen ion in the majority of these studies. Discussions on the lability and kinetics can be found in some of these works. ${ }^{55,62-66}$ In all of them different equilibration times (after each metal addition) have been used, usually from $10 \mathrm{~min}$ up to overnight, depending on the author, sample, and metal ion determination. It should be noted that in many papers complexing parameters were determined from $i_{\mathrm{p}}$ assuming inert complexes. This should be valid for those metal ions forming natural organic complexes with conditional stability constants higher to $10^{10}$, but for conditional stability constants below $10^{9}$ the complexes may not act as inert.

2.5.2. Stripping Chronopotentiometry (SCP). Stripping chronopotentiometry (SCP), also named potentiometric stripping analysis (PSA), has been recently reviewed in environmental analysis. ${ }^{67} \mathrm{SCP}$ is a two-step technique involving (i) the deposition (accumulation), where metal ions are reduced at a constant potential and fixed time (in a similar manner as in ASV), followed by (ii) the stripping, where the accumulated metal is reoxidized through the application of a constant stripping current $\left(i_{\mathrm{ox}}\right)$ in quiescent solution (Figure 4). In earlier studies of stripping potentiometry, introduced by Jagner and Graneli in $1976,{ }^{68}$ the metal was reoxidized from the working electrode through an oxidant present in solution (e.g., $\mathrm{Hg}(\mathrm{II}))$. Although this method is still applicable in marine samples, ${ }^{69}$ the progressive improvement of the instrumentation allowed that the constant oxidizing current has become the predominant oxidant form, because in this mode the signal is free from $\mathrm{Hg}(\mathrm{II})$ disturbance. ${ }^{71}$

The signal $E$ versus time $(t)$ obtained in the stripping step produces a wave-shaped curve for each stripped species, provided the oxidation potentials are sufficiently separated. The analytical parameter to be measured is the transition time required for reoxidation $(\tau)$, defined between two consecutive potential jumps. For an accurate determination of $\tau, E$ vs $t$ should be converted in $\mathrm{d} t / \mathrm{d} E$ vs $E$, and the peak area (which represents $\tau$ ) measured $^{72}$ (Figure 4 ). When a thin mercury film electrode is used in SCP, there is a rapid metal transport inside the thin film during the stripping, which means that measurements are performed almost always under conditions of complete depletion, where the total charge is quantified (reoxidation of all the metal previously reduced in the mercury is attained). For the hanging mercury drop electrode, the conditions prevailing during the stripping step can range from 
semi-infinite linear diffusion $\left(i_{\mathrm{ox}} \tau^{1 / 2}\right.$ constant $)$ to the complete depletion limit $\left(i_{\mathrm{ox}} \tau\right.$ constant, which is attained for sufficiently small values of $i_{\text {ox }}$ ). For experiments performed under conditions approaching complete depletion, $\tau$ is directly proportional to the amount of material accumulated, and so depletive SCP allows the determination of the "labile" metal fraction in a very simple way. ${ }^{72}$

Depletive SCP has significant advantages compared to ASV: the requirement for an excess of ligand during the stripping step is minimized because $\tau$ is independent of any peak broadening, raised by ligand deficiency at the electrode surface or heterogeneity of metal complexes. ${ }^{73}$ Another advantage of SCP is its application in nondeaerated solutions when a TMFE is used. The fact that the full depletion mode is always maintained when this electrode is used allows the use of much higher stripping currents, thus eliminating the oxygen interference. ${ }^{74}$ For other electrodes, like the hanging mercury drop electrode, an extensive purging is necessary to avoid the competition of the chemical stripping caused by oxygen traces in solution. Finally, from a theoretical point of view, the analytical signal $\tau$ should be practically free from induced metal adsorption. ${ }^{75}$ However, in complex media as marine waters, Louis et al. $^{76}$ showed that the determination of copper by stripping chronopotentiometry in depletive mode is influenced by the adsorbed layer in the same manner as in DPASV. They claim that introducing a desorption step just before stripping (in the same manner as for ASV) the adsorption effects vanished, yielding unaffected final results.

As for ASV, only metals forming amalgam can be determined by SCP on a mercury electrode. To extend the application to other metal ions, adsorptive stripping chronopotentiometry (AdSCP) has been considered as an alternative technique, following the same concept as in adsorptive cathodic stripping voltammetry. In AdSCP, a ligand $\left(\mathrm{L}_{\mathrm{ad}}\right)$ added to the solution forms with the metal ion a surface active complex $\left(\mathrm{ML}_{\mathrm{ad}}\right)$ that is adsorbed on the electrode. ${ }^{77}$ The metal ion is, then, quantified by the application of a reducing constant current. The theory underlying this technique has been developed very recently, and it was shown that a single adsorptive stripping measurement is only feasible under rather restrictive conditions. $^{78}$ No applications of AdSCP to marine waters have been found yet.

One limitation of SCP in the presence of ligands is the lack of a simple relationship between the peak potential $\left(E_{\mathrm{p}}\right)$ and the speciation in the sample solution. This is overcome using scanned stripping chronopotentiometry, a particularly powerful tool for dynamic trace metal speciation analysis. The potentialities/limitations of SSCP were reviewed by the same authors and compared with other stripping techniques. ${ }^{79}$

The SSCP curve is constructed from a series of individual depletive SCP curves measured at different deposition potentials $\left(E_{\mathrm{d}}\right)$, varying from values in the limiting current to the potential range where no further metal reduction occurs. Representing $\tau$ vs $E_{\mathrm{d}}$ a sigmoidal-shape curve is obtained (Figure 5). A similar kind of curves was previously obtained by ASV (called pseudopolarograms), plotting the stripping current as a function of the applied deposition potential. They were first used by Nurnberg, Branica, et al. ${ }^{80,81}$ to evaluate the complexation of trace metals in marine samples. The theory of SSCP for the determination of metal speciation parameters under different conditions has been developed by van Leeuwen and Town, and the main conclusions presented below. These authors show that careful assessment of the features of the

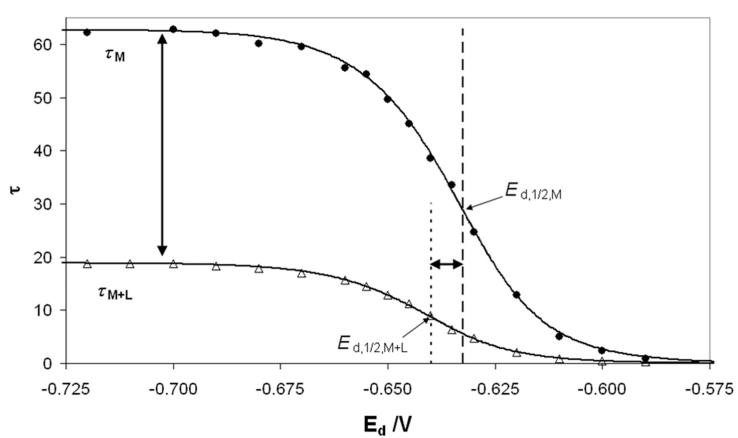

Figure 5. SSCP curves of $3 \times 10^{-7} \mathrm{M}$ cadmium in the absence ( and presence of $60 \mathrm{~nm}$ radius carboxylated latex spheres $(\Delta)$, at $\mathrm{pH}$ 6.0 and $0.1 \mathrm{~mol} \mathrm{~m}^{-3} \mathrm{KNO}_{3}$. Lines are fitted curves. $D_{\mathrm{cd}}=7 \times 10^{-10} \mathrm{~m}^{2}$ $\mathrm{s}^{-1}$. Experiment performed using a hanging mercury drop electrode with area $4 \times 10^{-7} \mathrm{~m}^{2}$ and volume $2.4 \times 10^{-11} \mathrm{~m}^{3}$, using a deposition time of $90 \mathrm{~s}$ and a stripping current of $10^{-9} \mathrm{~A}$.

SSCP waves can identify the degree of reversibility for nonreversible electron transfer reactions and in some cases allow metal complexation parameters to be determined in a straightforward manner. ${ }^{82}$ Intermetallic compound formation distorts the shape of the SSCP wave, with least impact under depletive SSCP conditions; systematic measurements over a range of conditions (oxidizing current, deposition time, potential deposition, electrode size) generally allow the identification of the processes occurring and of the best experimental conditions that minimize mixture effects. ${ }^{83}$ For labile complexes it was found that the shift in half-wave potential and the influence of the diffusion coefficient of metal complexes have the same meaning as in DeFord-Hume treatment of polarographic waves, ${ }^{84}$ providing a reliable measure of metal speciation in the presence of simple ligands. In addition, the SSCP approach is still valid when there is insufficient ligand excess at the electrode surface during the stripping step and should not be affected by induced metal adsorption, as commented before for depletive SCP. The presence of heterogeneous ligands, with a range of metal complexes of slightly different stability and mobility, turns the slope of SSCP wave less steep than in homogeneous case. ${ }^{85-88}$ An approach was developed to describe the SSCP curve for a heterogeneous sample under conditions where the deposition flux is limited by the rate of complex dissociation. ${ }^{89}$ The distribution of the dissociation rate constants $\left(k_{\mathrm{d}}\right)$ obtained from the SSCP wave is a basis for translation of speciation data into predictions of other dynamic processes such as biouptake, because the range of $k_{\mathrm{d}}$ values accessible by SSCP corresponds to processes on a time scale comparable to the metal biouptake by microorganisms and fish gills. ${ }^{89}$ Finally, the comparison of the stability constants obtained from the two SSCP signals $(\tau$ and $E_{\mathrm{d}, 1 / 2}$, Figure 5) provides information about the dynamic nature of the metal complexes with macromolecules and in colloidal dispersions, in reasonable agreement with the predictions from the dynamic theory (sections 2.3 and 2.4).

Detection limits in the range of $10^{-8} \mathrm{~mol} \mathrm{dm}^{-3}$ or even below make possible the quantification of trace elements by SCP at the low concentrations usually existing in environmental saline waters. The labile fraction of the analyte has been determined by SCP in those waters, which is very important in the point of view of toxicity and bioavailability. Still, there are no references on the fitting of experimental data to SSCP models in those waters, because only very recently SSCP theory 
applied to heterogeneous systems has been developed and validated for simpler systems. Therefore, this is an open field for future work in marine waters.

Some significant SCP applications have been found in environmental saline waters, mainly in the determination of the labile fraction of $\mathrm{Cu}^{90-92}$ and in the discrimination between As(III) and As(V). ${ }^{93-96}$

2.5.3. Competitive Ligand Exchange-Adsorptive Cathodic Stripping Voltammetry. Competitive ligand exchange-adsorptive cathodic stripping voltammetry (CLE-AdCSV) has been widely used to examine the speciation of trace metals in saline waters since the pioneer works of van den Berg. ${ }^{97}$ This technique is not restricted to metal ions that form amalgams with the electrode, and so it allows a much broader application than anodic stripping voltammetry.

In CLE-AdSV, a well-characterized ligand $\left(\mathrm{L}_{\mathrm{ad}}\right)$ of known competition strength is added to the solution and allowed to equilibrate with the metal-binding organic ligands present in the sample. Different typical added ligands and different periods of time, from some minutes to some hours, have been used in environmental saline waters.

After solution equilibration there is an adsorptive accumulation of the $\mathrm{ML}_{\mathrm{ad}}$ complex onto the electrode at an appropriated potential, and subsequent reduction of the adsorbed metal ion by means of a potential scanned to more negative potentials. The measured concentration is directly related to the $\mathrm{ML}_{\mathrm{ad}}$ concentration in the solution. If the effective stability constant of $\mathrm{ML}_{\mathrm{ad}}$ under the experimental conditions is known as well as $\mathrm{L}_{\mathrm{ad}}$ concentration $\left(\mathrm{L}_{\mathrm{ad}}\right.$ is added in excess so that the fraction not bound to $\mathrm{M}$ is equal to total ligand concentration), the free metal ion in solution and the metal fraction bound to natural ligands $\mathrm{L}$ can be determined. ${ }^{98-100}$ The stability constant $\mathrm{ML}$ and total ligand concentration has been determined from the titration of the solution with the metal ion followed by CLE-AdCSV. It is important to emphasize that, if the complexing strength of $\mathrm{L}_{\mathrm{ad}}$ is too high compared to that of natural ligands in solution, all the metal ions will be complexed to $\mathrm{L}_{\mathrm{ad}}$ and the voltammetric signal is related to the total metal concentration. On the other hand, if the complexing strength is too low, $\mathrm{L}_{\text {ad }}$ will not be able to compete with $\mathrm{L}$ and the signal is related to the free metal ion in natural sample (responsible for the $\mathrm{ML}_{\mathrm{ad}}$ fraction formed). Therefore, the complexes detected in heterogeneous samples are strongly dependent on the detection window of the method, ${ }^{100-102}$ defined by the stability constant and concentration of the added ligand. To obtain clear information about metal ion speciation in marine waters, a range of different analytical windows have been be applied to a given sample, either by changing the added ligand, ${ }^{101-103}$ or by simply changing $L_{a d}$ concentration, ${ }^{100,103,104}$ providing a continuum of values. Forward (metal ion addition) and reverse (CLE addition) titrations were both performed in some papers to check the coherence in the complexing parameters obtained ${ }^{104}$ or to determine strong ligands (not in excess) by reverse titration, in addition to those in excess. ${ }^{105}$ In other papers CLEAdCSV and ASV were applied to the same water samples for comparison. The different results obtained, where the weakest sites were determined by ASV, point to the complementary of these two techniques. ${ }^{101,103,106}$ To truly compare metal ion binding from different techniques as well as for different systems and metal ions, all the data should be obtained within the same detection window. ${ }^{107}$
Some kinetic limitations in the determination of trace metal speciation by CLE-AdCSV should be taken into consideration. Equilibration in solution should really be attained in the first step of the method so that CLE-AdCSV can be an equilibrium based method. Usually equilibrium is considered to be attained when, after $\mathrm{L}_{\text {ad }}$ addition, the measured current remains constant with time. However, the release of the metal ion from natural complexes with large $K_{\mathrm{ML}}$ and low dissociation rate constant may be sufficiently small, so that the change in the signal with time falls within the experimental error, leading to an apparent equilibrium. $^{108}$ Erroneous values will be then determined because kinetics must be considered. $\mathrm{L}_{\text {ad }}$ typically forms stable complexes with relatively fast formation rates, and so the limiting step in the equilibration process is the rate of $M L_{a d}$ dissociation. In this case the equilibrium time is given by $t_{\mathrm{eq}} \gg$ $1 / k_{\mathrm{d}} \cdot{ }^{108}$ The dissociation rate constant of $\mathrm{ML}_{\mathrm{ad}}\left(k_{\mathrm{d}}\right)$ can be estimated from the thermodynamic stability constant $(K)$ and the formation rate constant of the complex $\left(k_{\mathrm{a}}\right)$, through the relationship $K=k_{\mathrm{a}} / k_{\mathrm{d}}$. Assuming the Eigen mechanism (section 2.1 ), it can be seen that $t_{\mathrm{eq}}$ is most dependent on the metal ion through its $k_{\mathrm{w}}$. For $\mathrm{Ni}, \mathrm{Zn}, \mathrm{Pb}, \mathrm{Cu}$, and $\mathrm{Cd}, k_{\mathrm{w}}\left(\mathrm{s}^{-1}\right)$ is about 3 $\times 10^{4}, 7 \times 10^{7}, 7 \times 10^{9}, 1 \times 10^{9}$, and $3 \times 10^{8}$, respectively. ${ }^{10,108}$ For an ionic strength $0.1 \mathrm{~mol} \mathrm{dm}^{-3}$, ligand charge -2 and metal charge $+2, K^{\text {os }}$ will have an approximate value of $13{ }^{11}$ In this case and for $K_{\mathrm{ML}} \sim 10^{12}$, the equilibration time would be of months for $\mathrm{Ni}$, hours for $\mathrm{Zn}$, and minutes for $\mathrm{Pb}, \mathrm{Cu}$, or $\mathrm{Cd}$. Thus, equilibration times of about $12 \mathrm{~h}$ or below, as often found in experiments of CLE-AdCSV, are completely inadequate for $\mathrm{Ni}$ with $K_{\mathrm{ML}} \sim 10^{12}$, and an overestimated stability constant will be found from CLE-AdCSV measurements compared to real values. For equilibration times of about $12 \mathrm{~h}$, the stability constant $K_{\mathrm{ML}}$ of Ni should be lower than $10^{9}$.

Other kinetic limitations should be considered in the presence of weak complexation (determined when low detection windows are applied), because a kinetic contribution may also appear if $\mathrm{ML}$ dissociation/ $\mathrm{ML}_{\mathrm{ad}}$ association occur within the diffusion layer during the adsorption step. ${ }^{109}$ In this case underestimated $K_{\mathrm{ML}}$ values will be found. Concluding, metal speciation from CLE-AdCSV is valid if kinetics features are properly taken into account (e.g., waiting long enough).

The complexation of $\mathrm{Cu},{ }^{100-106} \mathrm{Zn},{ }^{98,110}$ and $\mathrm{Ni}^{111,112}$ in environmental saline waters have been determined by CLEAdCSV, as well as discrimination between As(III) and As $(\mathrm{V}){ }^{113,114}$ and between $\mathrm{Cr}(\mathrm{III})$ and $\mathrm{Cr}(\mathrm{CVI}){ }^{115,116}$

2.6. Perspectives and Future Trends in Trace Metal Complexation in Seawaters by Electrochemical Methods. The major difficulty in an overview of organic complexation in marine systems is due to the complexity of the medium and the heterogeneity of the ligands involved. The variety of electrochemical techniques, with different time scales and/or detection windows, may "see" different realities of the same heterogeneous sample, and so results should not be compared without a serious discussion on their meaning. Each dynamic technique has its characteristic lability criterion that determines the operationally dynamic metal species. ${ }^{9}$ Coupling the results obtained either from different techniques and/or from several analytical laboratories with shared samples, involving the widest possible variety of methods, seems essential for a correct data interpretation and system characterization. Large variations in organic-metal complexation have often been attributed to differences in natural samples characteristics rather than to differences due to different analytical procedures. ${ }^{59}$ 
Other important direction in the study of trace metal complexation in seawaters includes developments of voltammetric and chronopotentiometric measurements with low detection limits, high sensitivity and reliability. SSCP, although very promising, need to be further tested and validated in the presence of different natural saline waters and a more rigorous approach for SSCP speciation calculations in the presence of heterogeneous samples should be developed.

\section{AUTHOR INFORMATION}

\section{Notes}

The authors declare no competing financial interest.

\section{ACKNOWLEDGMENTS}

A.M.M./M.L.S.G. acknowledge the financial support of FCT and CQE/IST/Technical University of Lisbon and JPP acknowledges the financing received through FCT and IBB/ CBME, LA.

\section{REFERENCES}

(1) Florence, T. M. Analyst 1986, 111, 489-505.

(2) van Leeuwen, H. P.; Town, R.; Buffle, J.; Cleven, R. F.; Davison, W.; Puy, J.; Riemsdijk, W.; Sigg, L. Electroanal. 2001, 13, 826-830.

(3) van Leeuwen, H.; Town, R.; Buffle, J.; Cleven, R. F. M. J.; Davison, W.; Puy, J.; Riemsdijk, W. v.; Sigg, L. Environ. Sci. Technol. 2005, 39, 8545-8565.

(4) Campbell, P. G. C. Interactions between trace metals and aquatic organisms: a critique of the free-ion activity model. In Metal Speciation and Bioavailability in Aquatic Systems; Tessier, A., Turner, D. R., Eds.; John Wiley \& Sons: Chichester, U.K., 1995; Vol. 3.

(5) Slaveykova, V. I.; Wilkinson, K. J. Environ. Chem. 2005, 2, 9-24.

(6) van Leeuwen, H. P. Environ. Sci. Technol. 1999, 33, 3743-3748.

(7) de Jong, H. G.; van Leeuwen, H.; Holub, K. J. Electroanal. Chem. 1987, 234, 1-16.

(8) van Leeuwen, H. P.; Cleven, R.; Buffle, J. Pure Appl. Chem. 1989, $61,255-274$.

(9) Sigg, L.; Black, F.; Buffle, J.; Cao, J.; Cleven, R.; Davison, W.; Galceran, J.; Gunkel, P.; Kalis, E; Kistler, D.; Martin, M.; Noel, S.; Nur, Y.; Odzak, N.; Puy, J.; Riemsdijk, W. v.; Temminghoff, E.; TercierWaeber, M. L.; Toepperwien, S.; Town, R. M.; Unsworth, E.; Warnken, K. W.; Weng, L.; Xue, H.; Zhang, H. Environ. Sci. Technol. 2006, 40, 1934-1941.

(10) Eigen, E. Pure Appl. Chem. 1963, 6, 97-115.

(11) Margerum, D. W.; Cayley, G. R.; Weatherburn, D. C.; Pagenkofpf, G. K. Kinetics and mechanisms of complex formation and ligand exchange. In Coordination Chemistry; Martell, A., Ed.; ACS Monograph 174; American Chemical Society: Washington, DC, 1978; p 1.

(12) Fuoss, R. J. Am. Chem. Soc. 1958, 80, 5059-5061.

(13) Morel, F. M. M.; Hering, J. G. Principles and Applications of Aquatic Chemistry; Wiley: New York, 1993.

(14) Lyklema, J. Fundamentals of Interface and Colloid Science. Vol. II: Solid-Liquid Interfaces; Academic Press: London, 1995.

(15) Davison, W. J. Electroanal. Chem. 1978, 87, 395-404.

(16) Pinheiro, J.; Minor, M.; van Leeuwen, H. P. Langmuir 2005, 21, $8635-8642$.

(17) van Leeuwen, H. P.; Town, R. M.; Buffle, J. J. Phys. Chem. A 2007, 111, 2115-2121.

(18) Town, R. M.; van Leeuwen, H. P. J. Phys. Chem. A 2008, 112, $2563-2571$.

(19) van Leeuwen, H. P.; Town, R. M. Environ. Sci. Technol. 2009, 43, $88-93$.

(20) Buffle, J.; Zhang, Z.; Startchev, K. Environ. Sci. Technol. 2007, 41, $7609-7620$.

(21) van Leeuwen, H. P.; Buffle, J. Environ. Sci. Technol. 2009, 43, $7175-7183$.
(22) Buffle, J.; Zhang, Z.; Alemani, D. Environ. Sci. Technol. 2007, 41, $7621-7631$

(23) van Leeuwen, H. P. Langmuir 2008, 24, 11718-11721.

(24) Duval, J. F. L.; Pinheiro, J. P.; van Leeuwen, H. P. J. Phys. Chem. A 2008, 112, 7137-7151.

(25) Duval, J. F. L. J. Phys. Chem. A 2009, 113, 2275-2293.

(26) Duval, J. F. L.; Qian, S. J. Phys. Chem. A 2009, 113, 1279112804 .

(27) van Leeuwen, H. P.; Town, R. M.; Buffle, J. Langmuir 2011, 27, 4514-4519.

(28) van Leeuwen, H. P. J. Electroanal. Chem. 1979, 99, 93-102.

(29) Galceran, J.; Puy, J.; Salvador, J.; Cecilia, J.; van Leeuwen, H. P. J. Electroanal. Chem. 2001, 505, 85-94.

(30) Molina, A.; Martínez-Ortiz, F.; Laborda, E. Electrochem. Commun. 2009, 11, 562-567.

(31) Molina, A.; Martínez-Ortiz, F.; Laborda, E.; Puy, J. Phys. Chem. Chem. Phys. 2010, 12, 5396-5404.

(32) van Leeuwen, H. P.; Puy, J.; Galceran, J.; Cecilia, J. J. Electroanal. Chem. 2002, 526, 10-18.

(33) van Leeuwen, H. P.; Town, R. M. J. Electroanal. Chem. 2004, $561,67-74$

(34) Pinheiro, J. P.; van Leeuwen, H. P. J. Electroanal. Chem. 2004, 570, 69-75.

(35) Pinheiro, J. P.; Minor, M.; van Leeuwen, H. P. J. Electroanal. Chem. 2006, 587, 284-292.

(36) Pinheiro, J. P.; Domingos, R. F.; Minor, M.; van Leeuwen, H. P. J. Electroanal. Chem. 2006, 596, 57-64.

(37) Galceran, J.; Puy, J.; Salvador, J.; Cecilia, J.; Mas, F.; Garces, J. L. Phys. Chem. Chem. Phys. 2003, 5, 5091-5100.

(38) Puy, J.; Cecilia, J.; Galceran, J.; Town, R. M.; van Leeuwen, H. P. J. Electroanal. Chem. 2004, 571, 121-132.

(39) van Leeuwen, H. P.; Town, R. M. J. Electroanal. Chem. 2006, $587,148-154$.

(40) Salvador, J.; Puy, J.; Galceran, J.; Cecilia, J.; Town, R. M.; van Leeuwen, H. P. J. Phys. Chem. B 2006, 110, 891-899.

(41) Salvador, J.; Garces, J. L.; Galceran, J.; Puy, J. J. Phys. Chem. B 2006, 110, 13661-13669.

(42) Salvador, J.; Garces, J. L.; Companys, E.; Cecilia, J.; Galceran, J.; Puy, J.; Town, R. M. J. Phys. Chem. A 2007, 111, 4304-4311.

(43) Buffle, J.; Startcheva, K.; Galceran, J. Phys. Chem. Chem. Phys. 2007, 9, 2844-2885.

(44) Zhang, Z.; Buffle, J. J. Phys. Chem. A 2009, 113, 6562-6571.

(45) Zhang, Z.; Buffle, J.; Town, R. M.; Puy, J.; van Leeuwen, H. P. J. Phys. Chem. A 2009, 113, 6572-6580.

(46) Zhang, Z.; Buffle, J. Environ. Sci. Technol. 2009, 43, 5762-5768.

(47) Zhang, Z.; Alemani, D.; Buffle, J.; Town, R. M.; Wilkinson, K. J. Phys. Chem. Chem. Phys. 2011, 13, 17606-17614.

(48) Zhang, Z.; Buffle, J.; Startchev, K.; Alemani, D. Environ. Chem. 2008, 5, 204-217.

(49) Pinheiro, J. P.; Salvador, J.; Companys, E.; Galceran, J.; Puy, J. Phys. Chem. Chem. Phys. 2010, 12, 1131-1138.

(50) Buffle, J. Study of complexation properties by voltammetric methods. In Complexation Reactions in Aquatic Systems: an analytical approach; Ellis Horwood Pub.: Chichester, U.K., 1988.

(51) Pesavento, M.; Alberti, G.; Biesuz, R. Anal. Chim. Acta 2009, 631, 129-141.

(52) Tercier-Waeber, M. L.; Confalonieri, F.; Koudelka-Hep, M.; Dessureault-Rompre, J; Graziottin, F.; Buffle, J. Electroanal. 2008, 20, $240-258$

(53) Raspor, B.; Nürnberg, H. W.; Valenta, P.; Branica, M. J. Electroanal. Chem. 1980, 115, 293-308.

(54) Hering, J. G.; Morel, F. M. M. Environ. Sci. Technol. 1988, 22, $1469-1478$.

(55) Louis, Y.; Garnier, C.; Lenoble, V.; Mounier, S.; Cukrov, N.; Omanović, D.; Pižeta, I. Mar. Chem. 2009, 114, 110-119.

(56) Omanovic, D.; Garnier, C.; Louis, Y.; Lenoble, V.; Mounier, S.; Pizeta, I. Anal. Chim. Acta 2010, 664, 136-143.

(57) Mota, A. M.; Santos, M. C. Trace metal speciation of labile chemical species in natural waters: electrochemical methods. In Metal 
Speciation and Bioavailability in Aquatic Systems; Tessier, A., Turner, D. R., Eds.; John Wiley \& Sons: Chichester, U.K., 1995; Vol. 3.

(58) van Leeuwen, H. P. Sci. Total Environ. 1987, 60, 45-55.

(59) Byrne, R. Pure Appl. Chem. 1996, 68, 1639-1656.

(60) Florence, T. M.; Mann, K. J. Anal. Chim. Acta 1987, 200, 305312.

(61) Scarano, G.; Romei, C.; Seritti, A.; Zirino, A. Anal. Chim. Acta 1991, 245, 177-181.

(62) Muller, F. L. L.; Kester, D. R. Mar. Chem. 1991, 33, 71-90.

(63) Zirino, A.; Belli, S. L.; van der Weele, D. A. Electroanal. 1998, $10,423-427$.

(64) Fontes, C. L.; Mota, A. M.; Gonçalves, M. L. S. Water Res. 2000, 34, 3325-3334.

(65) Hurst, M. P.; Bruland, K. W. Anal. Chim. Acta 2005, 546, 6878.

(66) Jakuba, R. W.; Moffett, J. W.; Saito, M. A. Anal. Chim. Acta 2008, 614, 143-152.

(67) Serrano, N.; Diaz-Cruz, J. M.; Arino, C.; Esteban, M. Electroanal. 2007, 19, 2039-2049.

(68) Jagner, D.; Graneli, A. Anal. Chim. Acta 1976, 83, 19-26.

(69) Waeles, M.; Riso, R. D.; Maguer, J.-F.; Guillaud, J.-F.; Corre, P. L. J. Mar. Syst. 2008, 72, 358-365.

(70) Estela, J. M.; Tomas, C.; Cladera, A.; Cerda, V. Crit. Rev. Anal. Chem. 1995, 25, 91-141.

(71) Town, R. M. Anal. Chim. Acta 1998, 363, 31-43.

(72) Town, R. M.; van Leeuwen, H. P. J. Electroanal. Chem. 2001, $509,58-65$.

(73) Town, R. M.; van Leeuwen, H. P. J. Electroanal. Chem. 2002, $535,11-25$.

(74) Parat, C.; Schneider, A.; Castetbon, A.; Potin-Gautier, M. Anal. Chim. Acta 2011, 688, 156-162.

(75) Town, R. M.; van Leeuwen, H. P. J. Electroanal. Chem. 2002, $523,1-15$.

(76) Louis, Y.; Cmuk, P.; Omanovi, D.; Garnier, C.; Lenoble, V.; Mounier, S.; Pizeta, I. Anal. Chim. Acta 2008, 606, 37-44.

(77) Town, R. M.; van Leeuwen, H. P. J. Electroanal. Chem. 2007, 610, 17-27.

(78) Town, R. M. J. Electroanal. Chem. 2009, 637, 72-78.

(79) Town, R. M.; van Leeuwen, H. P. Electroanal. 2004, 16, 458471.

(80) Nürnberg, H.; Valenta, P.; Mart, L.; Raspor, B.; Sipos, L. Fresenius Z. Anal. Chem. 1976, 282, 357-367.

(81) Branica, M.; Novak, D. M.; Bubic, S. Croat. Chem. Acta 1977, 49, 539-547.

(82) Town, R. M.; Pinheiro, Jose P.; Domingos, R,; van Leeuwen, H. P. J. Electroanal. Chem. 2005, 580, 57-67.

(83) Town, R. M.; van Leeuwen, H. P. J. Electroanal. Chem. 2004, 573, 147-157.

(84) Town, R. M.; van Leeuwen, H. P. J. Electroanal. Chem. 2003, $541,51-65$.

(85) Town, R. M.; van Leeuwen, H. P. Aust. J. Chem. 2004, 57, 983992.

(86) van Leeuwen, H.; Town, R. M. Environ. Sci. Technol. 2003, 37, $3945-3952$.

(87) Domingos, R. F.; Lopez, R.; Pinheiro, J. P. Environ. Chem. 2008, $5,24-32$.

(88) Serrano, N.; Diaz-Cruz, J. M.; Arino, C.; Esteban, M.; Puy, J.; Companys, E.; Galceran, J.; Cecilia, J. J. Electroanal. Chem. 2007, 600, 275-284.

(89) Town, R. M. Environ. Sci. Technol. 2008, 42, 4014-4021.

(90) Blake, A. C.; Chadwick, D. B.; Zirino, A.; I, R.-D. Estuaries 2004, 27, 437-447.

(91) Daniele, S.; Bragato, C.; Baldo, M. A.; Wang, J.; Lu, J. Analyst 2000, 125, 731-735.

(92) Wang, J.; Larson, D.; Foster, N.; Armalis, S.; Lu, J.; Rongrong, X.; Olsen, K.; Zirino, A. Anal. Chem. 1995, 67, 1481-1485.

(93) La Pera, L.; Bella, G. D.; Rando, R.; Vincenzo, L. T.; Dugo, G. Environ. Monit. Assess. 2008, 145, 119-126.
(94) Salaun, P.; Planer-Friedrich, B.; van den Berg, C. M. G. Anal. Chim. Acta 2007, 585, 312-322.

(95) Vandenhecke, J.; Waeles, M.; Riso, R. D.; Corre, P. L. Anal. Bioanal. Chem. 2007, 388, 929-937.

(96) Huiliang, H.; Jagner, D.; Renman, L. Anal. Chim. Acta 1988, 207, 37-46.

(97) van den Berg, C. M. G. Anal. Chim. Acta 1991, 250, 265-276.

(98) Donat, J. R.; Bruland, K. W. Mar. Chem. 1990, 28, 301-323.

(99) Van den Berg, C. M. G. Mar. Chem. 1985, 16, 121-130.

(100) Buck, K. N.; Bruland, K. W. Mar. Chem. 2005, 96, 185-198.

(101) Bruland, K. W.; Rue, E. L.; Donat, J. R.; Skrabal, S. A.; Moffett, J. W. Anal. Chim. Acta 2000, 405, 99-113.

(102) van den Berg, C. M. G.; Donat, J. R. Anal. Chim. Acta 1992, 257, 281-291.

(103) Moffett, J. W.; Dupont, C. Deep-Sea Res. Pt. I 2007, 54, 586595.

(104) Nuester, J.; van den Berg, C. M. G. Anal. Chem. 2005, 77, 1119.

(105) Santos-Echeandía, J.; Laglera, L. M.; Prego, R.; van den Berg, C. M. G. Mar. Chem. 2008, 108, 148-158.

(106) Gerringa, L. J. A.; Poortvliet, T. C. W.; Hummel, H. Estuar. Coast. Shelf Sci. 1996, 42, 629-643.

(107) Town, R. M.; Filella, M. Anal. Chim. Acta 2002, 466, 285-293.

(108) van Leeuwen, H.; Town, R. Environ. Sci. Technol. 2005, 39, $7217-7225$

(109) van Leeuwen, H. P.; Jansen, S. J. Electroanal. Chem. 2005, 579, 337-342.

(110) Ellwood, M. J.; van den Berg, C. M. G. Mar. Chem. 2000, 68, 295-306.

(111) Turner, A.; Nimmo, M.; Thuresson, K. A. Mar. Chem. 1998, 63, $105-118$.

(112) Martino, M.; Turner, A.; Nimmo, M. Mar. Chem. 2004, 88, 161-177.

(113) Sander, S.; Koschinsky, A. Mar. Chem. 2000, 71, 83-102.

(114) Boussemart, M.; van den Berg, C. M. G.; Ghaddaf, M. Anal. Chim. Acta 1992, 262, 103-115.

(115) Zima, J.; van den Berg, C. M. G. Anal. Chim. Acta 1994, 289, 291-298.

(116) de Carvalho, L. M.; Nascimento, P. C.; Bohrer, D.; Stefanello, R.; Pilau, E. J.; Rosa, M. B. Electroanalysis 2008, 20, 776-781. 